\title{
UNIVERSALLY CATENARIAN DOMAINS OF $D+M$ TYPE
}

\author{
DAVID F. ANDERSON, DAVID E. DOBBS, SALAH KABBAJ AND S. B. MULAY
}

(Communicated by Louis J. Ratliff, Jr.)

\begin{abstract}
Let $T$ be a domain of the form $K+M$, where $K$ is a field and $M$ is a maximal ideal of $T$. Let $D$ be a subring of $K$ and let $R=D+M$. It is proved that if $K$ is algebraic over $D$ and both $D$ and $T$ are universally catenarian, then $R$ is universally catenarian. The converse holds if $K$ is the quotient field of $D$. As a consequence, we construct for each $n>2$, an $n$-dimensional universally catenarian domain which does not belong to any previously known class of universally catenárian domains.
\end{abstract}

1. Introduction. All rings considered below are (commutative integral) domains. A ring $A$ is said to be catenarian if, for each pair $P \subset Q$ of prime ideals of $A$, all saturated chains of primes from $P$ to $Q$ have a common finite length. Following [3], we say that $A$ is universally catenarian if the polynomial rings $A\left[X_{1}, \ldots, X_{n}\right]$ are catenarian for each positive integer $n$. The main purpose of this note is to construct new examples of universally catenarian domains.

Any Cohen-Macaulay ring is universally catenarian. Moreover, it is known [15, (2.6)] that a Noetherian ring $A$ is universally catenarian if (and only if) $A[X]$ is catenarian. Moving beyond the Noetherian context, note that each catenarian $A$ must be locally finite-dimensional (LFD), in the sense that each prime ideal of $A$ has finite height. It is known $[\mathbf{1 4}, \mathbf{1 2}$, p. 256, 5] that each LFD Prüfer domain is universally catenarian. More generally, it was shown in [4, Theorem 1] that each LFD going-down strong $S$-domain (in the sense of [12]) must be universally catenarian. In addition, [4, Theorem 2] established that each LFD domain of global dimension 2 is universally catenarian. (As explained in [4, pp. 863-864], this assertion does not carry over to global dimension 3.) In $\S 3$, we construct for each integer $n \geq 2$, an $n$-dimensional universally catenarian domain which is not of any of the above types.

The constructions in $\S 3$ depend on work in $\S 2$ that studies universal catenarity for rings of the form $D+M$. Here, $M$ is a maximal ideal of a ring $K+M$, where $K$ is a field and $D$ is a subring of $K$. Theorem 2.2 characterizes universal catenarity in case $K$ is the quotient field of $D$. A useful sufficient condition is given in Corollary 2.3 , and Corollary 2.4 characterizes universal catenarity for the classsical $D+M$ construction [10] in which $K+M$ is a valuation domain.

Received by the editors October 5, 1987. Presented to the Society, March 25, 1988 (Knoxville, Tennessee).

1980 Mathematics Subject Classification (1985 Revision). Primary 13C15, 13G05; Secondary 13B25, 13A17, 13F05, 13D05, 13E05, 13B30, 14A05, $12 \mathrm{~F} 05$.

The third author was supported in part by NATO Collaborative Research Grant GR. 85/0035 and the University of Tennessee Science Alliance. He thanks the University of Tennessee for its warm hospitality during July-October, 1987. 
2. Universal catenarity and the $D+M$ construction. We begin with a useful result that is analogous to various gluing criteria in $[\mathbf{5}, \mathbf{4}, \mathbf{1}]$. It will be convenient to say that $I$ is $S$-saturated if $S$ is a multiplicatively closed subset of a ring $A$ and $I$ is an ideal of $A$ such that $A \cap S^{-1} I=I$. Note that if $I$ is an $S$-saturated proper ideal, then $I \cap S=\varnothing$.

LEMMA 2.1. Let $S$ be a multiplicatively closed subset of a domain $A$ and $I$ an $S$-saturated ideal of $A$. Let $P$ be a prime ideal of $A$ which contains $I$. Then there exists $Q$ in $\operatorname{Spec}(A)$ such that $I \subset Q \subset P$ and $Q \cap S=\varnothing$.

ProOF. We claim that $I A_{P}$ is an $S$-saturated ideal of $A_{P}$; in other words, if $u \in A_{P} \cap S^{-1} I A_{P}$, then $u \in I A_{P}$. To see this, note that there exists $z \in A \backslash P$ such that $z u \in A \cap S^{-1} I$. By hypothesis, $z u \in I$. Hence, $u=(z u) z^{-1} \in I A_{P}$, as claimed.

By the above comment, it follows that $I A_{P} \cap S=\varnothing$. Hence (cf. [10, Lemma 2.5]), there exists a prime ideal $W$ of $A_{P}$ such that $I A_{P} \subset W$ and $W \cap S=\varnothing$. Then $Q=W \cap A$ has the asserted properties.

We next set up riding hypotheses and notation for the rest of $\S 2$. Let $T$ be a domain of the form $K+M$, where $K$ is a field and $M$ is a (nonzero) maximal ideal of $T$. Let $D$ be a subring of $K$. Let $k$ be the quotient field of $D$ (inside $K$ ) and let $R=D+M$.

We are interested in knowing when $R$ is universally catenarian. The next result answers this completely in case $k=K$.

THEOREM 2.2. Suppose that $K$ is the quotient field of $D$. Then $R$ is universally catenarian if and only if both $T$ and $D$ are universally catenarian.

PROOF. The "only if" assertion follows from the fact that the class of universally catenarian domains is closed under localization and factor domains [3, Corollary 3.3]. The point is that $R / M \cong D$; and, if $S=D \backslash\{0\}$, then $S^{-1} R=k+M=$ $K+M=T$.

Conversely, suppose that both $T$ and $D$ are universally catenarian. Hence, both are LFD. We claim that $R$ is LFD. To see this, note first that $R$ is the pullback of the inclusion map $D \rightarrow K$ and the canonical projection $T \rightarrow K$. Accordingly, by [9, Theorem 1.4], $\operatorname{Spec}(R)$ can be characterized up to homeomorphism. The order-theoretic upshot is that, as a poset, $\operatorname{Spec}(R)$ is obtained by "gluing" $\operatorname{Spec}(D)$ onto $\operatorname{Spec}(T)$ in such a way that $\{0\} \in \operatorname{Spec}(D)$ coincides with $M \in \operatorname{Spec}(T)$. In particular, $R$ is LFD.

It follows that $A=R\left[X_{1}, \ldots, X_{n}\right]$ is also LFD for each positive integer $n$. To prove that $A$ is catenarian, we consider $P_{0} \subset \cdots \subset P_{s}=P$, any saturated chain of $s+1$ distinct primes in $A$.

Suppose $M\left[X_{1}, \ldots, X_{n}\right] \subset P_{0}$. It will suffice to show $\operatorname{ht}\left(P / P_{0}\right)=s$. Note that

$$
P_{0} / M\left[X_{1}, \ldots, X_{n}\right] \subset \cdots \subset P_{s} / M\left[X_{1}, \ldots, X_{n}\right]
$$

is a saturated chain of distinct primes in the catenarian domain $A / M\left[X_{1}, \ldots, X_{n}\right] \cong$ $D\left[X_{1}, \ldots, X_{n}\right]$. Now, it is easy to see that if $J_{1} \subset J_{2}$ are primes of a catenarian domain, then ht $\left(J_{2}\right)-\operatorname{ht}\left(J_{1}\right)=\operatorname{ht}\left(J_{2} / J_{1}\right)$. Thus,

$$
\operatorname{ht}\left(P / M\left[X_{1}, \ldots, X_{n}\right]\right)-\operatorname{ht}\left(P_{0} / M\left[X_{1}, \ldots, X_{n}\right]\right)=\operatorname{ht}\left(P / P_{0}\right) .
$$


Since

$$
\operatorname{ht}\left(P / M\left[X_{1}, \ldots, X_{n}\right]\right)=\operatorname{ht}\left(P_{0} / M\left[X_{1}, \ldots, X_{n}\right]\right)+s,
$$

we conclude that $\mathrm{ht}\left(P / P_{0}\right)=s$, as required in this case.

Suppose next that $M\left[X_{1}, \ldots, X_{n}\right] \not \subset P_{0}$. It will suffice to show $\operatorname{ht}(P)-\operatorname{ht}\left(P_{0}\right)=$ $s$. Let $S=D \backslash\{0\}$. We claim that $P_{0}$ is $S$-saturated. Indeed, consider $u \in$ $A \cap S^{-1} P_{0}$; we shall show that $u \in P_{0}$. Choose $z \in S$ such that $z u \in P_{0}$. As $z$ is a unit in $T$, we have $z M=M$, whence $u M\left[X_{1}, \ldots, X_{n}\right]=z u M\left[X_{1}, \ldots, X_{n}\right]$ is contained in $P_{0}$. Since $P_{0}$ is prime, it follows that $u \in P_{0}$, as claimed.

Since $P_{0}$ is $S$-saturated, $P_{0} \cap S=\varnothing$. There exists an integer $r$ such that $0 \leq r \leq s, P_{r} \cap S=\varnothing$ and, if $r<s$, then $P_{r+1} \cap S \neq \varnothing$. Defining

$$
I=P_{r}+M\left[X_{1}, \ldots, X_{n}\right],
$$

we have

$$
S^{-1} I=S^{-1} P_{r}+S^{-1} M\left[X_{1}, \ldots, X_{n}\right]=S^{-1} P_{r}+M\left[X_{1}, \ldots, X_{n}\right] .
$$

Thus,

$$
A \cap S^{-1} I=\left(A \cap S^{-1} P_{r}\right)+M\left[X_{1}, \ldots, X_{n}\right]=P_{r}+M\left[X_{1}, \ldots, X_{n}\right]=I ;
$$

that is, $I$ is an $S$-saturated ideal of $A$.

Suppose, for the moment, that $r<s$. Pick $d \in P_{r+1} \cap S$ and observe that

$$
M=d\left(d^{-1} M\right) \subset d M \subset P_{r+1} .
$$

It follows that $P_{r+1}$ contains $I$. Hence, Lemma 2.1 may be applied, yielding $Q \in$ $\operatorname{Spec}(A)$ such that $I \subset Q \subset P_{r+1}$ and $Q \cap S=\varnothing$. As $Q \neq P_{r+1}$ and $h t\left(P_{r+1} / P_{r}\right)=$ 1 , we have $Q=P_{r}$. In particular, $P_{r}$ contains $M\left[X_{1}, \ldots, X_{n}\right]$. Viewing the chain induced in the catenarian domain $A / M\left[X_{1}, \ldots, X_{n}\right] \cong D\left[X_{1}, \ldots, X_{n}\right]$, we conclude that

$$
\operatorname{ht}\left(P / M\left[X_{1}, \ldots, X_{n}\right]\right)-\operatorname{ht}\left(P_{r} / M\left[X_{1}, \ldots, X_{n}\right]\right)=s-r .
$$

We claim that ht $(P)-\mathrm{ht}\left(P_{r}\right)=s-r$. By the above comments, it suffices to show

$$
\operatorname{ht}(P)-\operatorname{ht}\left(P_{r}\right)=\operatorname{ht}\left(P / M\left[X_{1}, \ldots, X_{n}\right]\right)-\operatorname{ht}\left(P_{r} / M\left[X_{1}, \ldots, X_{n}\right]\right) .
$$

Setting $p=P \cap R$ and $p_{r}=P_{r} \cap R$, we infer from [10, Theorem 30.18] that

$$
\operatorname{ht}(P)=\operatorname{ht}\left(p\left[X_{1}, \ldots, X_{n}\right]\right)+\operatorname{ht}\left(P / p\left[X_{1}, \ldots, X_{n}\right]\right)
$$

and

$$
\operatorname{ht}\left(P_{r}\right)=\operatorname{ht}\left(p_{r}\left[X_{1}, \ldots, X_{n}\right]\right)+\operatorname{ht}\left(P_{r} / p_{r}\left[X_{1}, \ldots, X_{n}\right]\right) .
$$

Now, viewing $R$ as the pullback of $D \rightarrow K$ and $T \rightarrow K$, we see via [1, Corollary 2.12] that $R$ is a locally Jaffard domain, in the sense of [1]. It follows that $h t(p)=$ $\operatorname{ht}\left(p\left[X_{1}, \ldots, X_{n}\right]\right)$, with a similar assertion for ht $\left(p_{r}\right)$. Hence, by the three previously displayed equations, the claim will follow if we show

$$
\begin{aligned}
\operatorname{ht}(p) & -\operatorname{ht}\left(p_{r}\right)+\operatorname{ht}\left(P / p\left[X_{1}, \ldots, X_{n}\right]\right)-\operatorname{ht}\left(P_{r} / p_{r}\left[X_{1}, \ldots, X_{n}\right]\right) \\
& =\operatorname{ht}\left(P / M\left[X_{1}, \ldots, X_{n}\right]\right)-\operatorname{ht}\left(P_{r} / M\left[X_{1}, \ldots, X_{n}\right]\right) .
\end{aligned}
$$

Now, since $A / M\left[X_{1}, \ldots, X_{n}\right] \cong D\left[X_{1}, \ldots, X_{n}\right]$ is catenarian,

$$
\begin{aligned}
& \operatorname{ht}\left(P / p\left[X_{1}, \ldots, X_{n}\right]\right)=\operatorname{ht}\left(\left(P / M\left[X_{1}, \ldots, X_{n}\right]\right) /\left(p\left[X_{1}, \ldots, X_{n}\right] / M\left[X_{1}, \ldots, X_{n}\right]\right)\right) \\
& \quad=\operatorname{ht}\left(P / M\left[X_{1}, \ldots, X_{n}\right]\right)-\operatorname{ht}\left(p\left[X_{1}, \ldots, X_{n}\right] / M\left[X_{1}, \ldots, X_{n}\right]\right) .
\end{aligned}
$$


A similar rewriting of $\mathrm{ht}\left(P_{r} / p_{r}\left[X_{1}, \ldots, X_{n}\right]\right)$ is possible.

Moreover, $p\left[X_{1}, \ldots, X_{n}\right] / M\left[X_{1}, \ldots, X_{n}\right]$ may be viewed as $(p / M)\left[X_{1}, \ldots, X_{n}\right]$ in the (universally catenarian, hence stably strong $S-$ ) domain $D\left[X_{1}, \ldots, X_{n}\right]$. Thus, ht $\left(p\left[X_{1}, \ldots, X_{n}\right] / M\left[X_{1}, \ldots, X_{n}\right]\right)=\operatorname{ht}(p / M)$; a similar assertion holds with $p_{r}$ replacing $p$. Hence, the claim will follow if we show

$$
\begin{aligned}
\operatorname{ht}(p)- & \operatorname{ht}\left(p_{r}\right)+\left(\operatorname{ht}\left(P / M\left[X_{1}, \ldots, X_{n}\right]\right)-\operatorname{ht}(p / M)\right) \\
& -\left(\operatorname{ht}\left(P_{r} / M\left[X_{1}, \ldots, X_{n}\right]\right)-\operatorname{ht}\left(p_{r} / M\right)\right) \\
& =\operatorname{ht}\left(P / M\left[X_{1}, \ldots, X_{n}\right]\right)-\operatorname{ht}\left(P_{r} / M\left[X_{1}, \ldots, X_{n}\right]\right) .
\end{aligned}
$$

This, in turn, will follow if $\operatorname{ht}(p / M)=\operatorname{ht}(p)-\operatorname{ht}(M)$ and $\operatorname{ht}\left(p_{r} / M\right)=\operatorname{ht}\left(p_{r}\right)-$ ht $(M)$. But these equations do hold. The reason is that $R$ is catenarian. Since $T$ and $D$ are catenarian, this fact follows from the earlier order-theoretic description of $\operatorname{Spec}(R)$. The upshot is that we have proved the claim, ht $(P)-\operatorname{ht}\left(P_{r}\right)=s-r$, in case $r<s$. Since this equation reduces to $0=0$ in case $r=s$, the claim has been established.

Finally, consider the saturated chain $S^{-1} P_{0} \subset \cdots \subset S^{-1} P_{r}$ of distinct primes in the catenarian domain $S^{-1} A=\left(S^{-1} R\right)\left[X_{1}, \ldots, X_{n}\right]=T\left[X_{1}, \ldots, X_{n}\right]$. We have

$$
\operatorname{ht}\left(P_{r}\right)-\operatorname{ht}\left(P_{0}\right)=\operatorname{ht}\left(S^{-1} P_{r}\right)-\operatorname{ht}\left(S^{-1} P_{0}\right)=\operatorname{ht}\left(S^{-1} P_{r} / S^{-1} P_{0}\right)=r \text {. }
$$

Hence,

$$
\operatorname{ht}(P)-\operatorname{ht}\left(P_{0}\right)=\left(\operatorname{ht}\left(P_{r}\right)-\operatorname{ht}\left(P_{0}\right)\right)+\left(\operatorname{ht}(P)-\operatorname{ht}\left(P_{r}\right)\right)=r+(s-r)=s .
$$

COROLLARY 2.3. If $T$ and $D$ are both universally catenarian and if $K$ is algebraic over $D$, then $R$ is universally catenarian.

PROOF. It will suffice to show that $k+M$ is universally catenarian. Indeed, since $M$ is a maximal ideal of $k+M$ and $D$ is universally catenarian, the conclusion will then follow from the "if" assertion in Theorem 2.2. Thus, we may assume that $D=k$ is a field. By hypothesis, $K$ is algebraic over $k$, and so $T$ is integral over $R$. Hence, to show that $R$ is universally catenarian, $[3$, Theorem 6.1] shows that it suffices to prove $\mathrm{ht}\left(q_{1}\right)=\operatorname{ht}\left(q_{2}\right)$ whenever $q_{1}, q_{2} \in \operatorname{Spec}(T)$ satisfy $q_{1} \cap R=$ $q_{2} \cap R$. However, this holds (indeed, $q_{1}=q_{2}$ ) since pullback considerations, using $[9$, Theorem 1.4] as in the proof of Theorem 2.2, yield that $\operatorname{Spec}(T) \rightarrow \operatorname{Spec}(R)$ is a homeomorphism.

COROLlARY 2.4. Suppose that $T$ is a finite-dimensional valuation domain which is not a field and that $D$ is finite-dimensional. Then $R$ is universally catenarian if and only if $D$ is universally catenarian and $K$ is algebraic over $D$.

PROOF. Since $T$ is an LFD Prüfer domain, $T$ is universally catenarian, by results recalled in $\S 1$. The "if" assertion is therefore a special case of Corollary 2.3. Conversely, suppose that $R$ is universally catenarian. Then so is $R / M \cong D$, by [3, Corollary 3.3]. By universal catenarity, [3, Corollary 3.3] shows that the valuative dimension of $R$ (resp., $D$; resp., $T$ ) coincides with its (Krull) dimension. Viewing $R$ as the pullback of $D \rightarrow K$ and $T \rightarrow K$, we may thus infer from [1, Theorem 2.6] that $\operatorname{dim}(R)=\operatorname{dim}(D)+\operatorname{dim}(T)+\mathrm{t} . \mathrm{d} .(K / k)$. However, it is well known (cf. [10, Exercise 12(4), pp. 202-203]) that $\operatorname{dim}(R)=\operatorname{dim}(D)+\operatorname{dim}(T)$. Hence, t. d. $(K / k)=0$; that is, $K$ is algebraic over $D$. 
We do not know if the converse of Corollary 2.3 is valid. We shall close this section with some remarks in this regard.

REMARK 2.5. (a) Suppose that $R$ is universally catenarian. Then, by [3, Corollary 3.3], so are $D$ and $k+M$; and the valuative dimension of $R$ (resp., $D$ ) coincides with its dimension. Now, if $R$ is also assumed finite-dimensional and if $T$ is quasilocal but not a field, various pullback results [1, Lemma 2.1(d) and Theorem 2.6(a) ] yield that $K$ is algebraic over $D$ (that is, over $k$ ).

(b) If either $k+M$ or $T$ is catenarian, then so is the other. This follows from the order-isomorphism $\operatorname{Spec}(T) \rightarrow \operatorname{Spec}(k+M)$ : see [9, Theorem 1.4].

(c) Assume that $D=k, K / k$ is algebraic, and $A=R\left[X_{1}, \ldots, X_{n}\right]$, where $R$ is universally catenarian. With $S=k\left[X_{1}, \ldots, X_{n}\right] \backslash\{0\}$, we have that $S^{-1} A=$ $k\left(X_{1}, \ldots, X_{n}\right)+S^{-1} M\left[X_{1}, \ldots, X_{n}\right]$ is universally catenarian. Since $K$ is algebraic over $k, B=T\left[X_{1}, \ldots, X_{n}\right]$ satisfies $S^{-1} B=K\left(X_{1}, \ldots, X_{n}\right)+S^{-1} M\left[X_{1}, \ldots, X_{n}\right]$. By applying (b), we infer that $S^{-1} B$ is catenarian. It follows, via [9, Theorem 1.4] as in the proof of Theorem 2.2 , that $K\left[X_{1}, \ldots, X_{n}\right]+S^{-1} M\left[X_{1}, \ldots, X_{n}\right]$ is catenarian. For this reason, we suggest that this ring's relation to $T\left[X_{1}, \ldots, X_{n}\right]$ merits closer attention.

We are also led to raise the following question. Let $A=E \oplus P$ be a domain, where $E$ is a subring and $P \in \operatorname{Spec}(A)$. Let $S$ denote $E \backslash\{0\}$ and assume that $B=E+S^{-1} P$ is catenarian. Under what conditions is $A$ catenarian?

Note that some conditions need to be imposed, for $A$ need not be catenarian in general. For instance, take $E$ to be a catenarian domain such that $E[X]$ is not catenarian, as in [13, Example 2, p. 203]. Put $P=X E[X]$ and let $L$ denote the quotient field of $E$. Then, since $L[X]$ and $E$ are catenarian, one may use [9, Theorem 1.4] as in the proof of Theorem 2.2 to conclude that $B=E+S^{-1} P=$ $E+X L[X]$ is catenarian. However, in this example, $A=E+P=E[X]$ is not catenarian.

(d) Suppose that $R$ is universally catenarian. In studying whether $T$ must be universally catenarian, we may assume that $D=k$ is a field (since, by (a), $k+M$ is universally catenarian). We claim that if the field extension $K / k$ is (algebraic) purely inseparable, then $T$ is universally catenarian.

For a proof, note first that $T(=K+M)$ is integral over $R(=k+M)$. Indeed, since $K / k$ is purely inseparable and $K M \subset M$, each element of $T$ has a power in $R$. This property is inherited by the extension $R\left[X_{1}, \ldots, X_{n}\right] \subset T\left[X_{1}, \ldots, X_{n}\right]$. Hence, for all $n \geq 1, \operatorname{Spec}\left(R\left[X_{1}, \ldots, X_{n}\right]\right)$ and $\operatorname{Spec}\left(T\left[X_{1}, \ldots, X_{n}\right]\right)$ are homeomorphic, and therefore order-isomorphic. (This can also be seen by showing that $T$ is the weak normalization of $R$ in $T$ and using the fact that weak normalization is a universal homeomorphism [2, Teorema 1].) In particular, $T\left[X_{1}, \ldots, X_{n}\right]$ is catenarian, proving the claim.

(e) Suppose that $(T, M)$ is quasilocal and finite-dimensional. Under these conditions, we may use (a) and (d) to reduce the converse of Corollary 2.3 to the following question. If $k+M$ is universally catenarian and the field extension $K / k$ is separable, is $T=K+M$ necessarily universally catenarian?

3. Examples. In this section, we apply Corollary 2.4 to construct the new family of universally catenarian domains promised in $\S 1$.

EXAMPLE 3.1. For each integer $n>2$, there exists an $n$-dimensional nonNoetherian universally catenarian domain $R_{n}$ such that $\operatorname{gl} \operatorname{dim}\left(R_{n}\right)>2$ and $R_{n}$ 
is neither a going-down strong $S$-domain nor a polynomial ring over a universally catenarian domain.

PROOF. Let $k$ be a field and take $n$ indeterminates $X_{1}, \ldots, X_{n-1}, Y$ over $k$. Consider the discrete (rank 1) valuation ring $V_{n}=k\left(X_{1}, \ldots, X_{n-1}\right)[Y]_{(Y)}=K_{n}+$ $M_{n}$, where $K_{n}=k\left(X_{1}, \ldots, X_{n-1}\right)$ and $M_{n}=Y V_{n}$. Put $R_{n}=D_{n}+M_{n}$, where $D_{n}=k\left[X_{1}, \ldots, X_{n-1}\right]$. Then, by well-known properties of the classical $D+M$ construction, $\operatorname{dim}\left(R_{n}\right)=\operatorname{dim}\left(D_{n}\right)+\operatorname{dim}\left(V_{n}\right)=(n-1)+1=n$ (cf. [10, Exercise 12(4), pp. 202-203]); and $R_{n}$ is not Noetherian (cf. [10, Exercise 8(3), pp. 270$271]$ ). As $D_{n}$ is universally catenarian (because $k$ is), Corollary 2.4 yields that $R_{n}$ is also universally catenarian.

Note that gl. $\operatorname{dim}\left(V_{n}\right)=1$ and $\operatorname{gl} \cdot \operatorname{dim}\left(D_{n}\right)=n-1$. Hence, by [7, Proposition 2.1(1)], we have that gl. $\operatorname{dim}\left(R_{n}\right)=n-1$ if p.d. $D_{n}\left(K_{n}\right)<n-1$, and $\operatorname{gl} . \operatorname{dim}\left(R_{n}\right)=n$ if p.d. $D_{n}\left(K_{n}\right)=n-1$. Thus, gl. $\operatorname{dim}\left(R_{n}\right)>2$ if $n>3$. For the case $n=3$, we must choose $k$ more carefully: take $k$ to be any uncountable field, for instance $\mathbf{R}$. Then [11, Theorem 2] assures that p. d. $_{D_{3}}\left(K_{3}\right)=2$, and so the above consequence of [7] yields gl. $\operatorname{dim}\left(R_{3}\right)=3$. Hence, for all $n>2, \operatorname{gl} \cdot \operatorname{dim}\left(R_{n}\right)>2$.

In $\operatorname{Spec}\left(R_{n}\right), X_{1} D_{n}+M_{n}$ and $X_{2} D_{n}+M_{n}$ are incomparable prime ideals contained in the maximal ideal $\left(X_{1}, \ldots, X_{n-1}\right)+M_{n}$. Thus $R_{n}$ is not treed; hence by [6, Theorem 2.2], $R_{n}$ is not a going-down (strong $S-$ ) domain. Finally, $R_{n}$ is not a polynomial ring because it has a unique height 1 prime ideal, namely $M_{n}$.

REMARK 3.2. (a) Note that, for the above construction, it is necessary to assume $n>2$. Indeed, any one-dimensional domain is a going-down domain; and, by $[8$, Corollary], so is any two-dimensional domain that is constructed via the classical $D+M$ construction.

(b) It remains to discuss the possibility of "new" examples for dimensions 1 and 2. In dimension $1,[3$, Corollary 6.3$]$ or $[4$, Theorem 1$]$ tells the whole story: a onedimensional domain is universally catenarian if and only if it is a strong $S$-domain.

As for dimension 2 , let $(V, M)$ be a non-Noetherian one-dimensional valuation domain. Then $A=V[X]_{(M, X)}$ is a two-dimensional non-Noetherian universally catenarian domain, of global dimension at least 3 , such that $A$ is neither a goingdown (strong $S-$ ) domain nor a polynomial ring (over a universally catenarian domain).

We shall make only three comments by way of proof, with the rest of the verification left to the reader. If $\left\{I_{i}\right\}$ is a strictly ascending chain of ideals in $V$, then $\left\{I_{i} A\right\}$ is also strictly ascending, and so $A$ is non-Noetherian. If $a$ and $b$ are nonassociated elements of $M$ in $V$, then a calculation shows $(X+a)(X+b)^{-1} \notin A$, and so $A$ is not a valuation domain. Since $A$ is integrally closed and has valuative dimension 2, it follows from [6, Proposition 2.7] that $A$ is not a going-down domain.

(c) The construction in (b) generalizes to give another new family of examples, as follows. Let $n \geq 2$ and $(V, M)$ be an $(n-1)$-dimensional valuation domain. Assume that $V_{P}$ is not Noetherian, where $P$ is the unique height 1 prime of $V$. Then $A=V[X]_{(M, X)}$ is an $n$-dimensional non-Noetherian universally catenarian domain, of global dimension at least 3 , such that $A$ is neither a going-down domain nor a polynomial ring.

The proof is nearly the same as in (b), with the following exception. To see that gl. $\operatorname{dim}(A)>2$, note that $B=V[X]_{(P, X)}=V_{P}[X]_{\left(P_{P}, X\right)}$ is a localization of $A$ and gl. $\operatorname{dim}(B)>2$ by (b). 


\section{REFERENCES}

1. D. F. Anderson, A. Bouvier, D. E. Dobbs, M. Fontana and S. Kabbaj, On Jaffard domains, Exposition. Math. 6 (1988), 145-175.

2. A. Andreotti and E. Bombieri, Sugli omeomorfismi delle varietà algebriche, Ann. Scuola Norm. Sup. Pisa 23 (1969), 431-450.

3. A. Bouvier, D. E. Dobbs and M. Fontana, Universally catenarian integral domains, Adv. in Math. (to appear).

4. __ Two sufficient conditions for universal catenarity, Comm. Algebra 15 (1987), 861-872.

5. A. Bouvier and M. Fontana, The catenarian property of polynomial rings over a Prüfer domain, Sém. Algèbre P. Dubreil et M. Malliavin, Lecture Notes in Math., vol. 1146, Springer-Verlag, Berlin and New York, 1985, pp. 340-354.

6. D. E. Dobbs, On going-down for simple overrings. II, Comm. Algebra 1 (1974), 439-458.

7. $\ldots$, On the global dimensions of $D+M$, Canad. Math. Bull. 18 (1975), 657-660.

8. D. E. Dobbs and I. J. Papick, On going-down for simple overrings. III, Proc. Amer. Math. Soc. 54 (1976), 35-38.

9. M. Fontana, Topologically defined classes of commutative rings, Ann. Mat. Pura Appl. 123 (1980), 331-355.

10. R. Gilmer, Multiplicative ideal theory, Dekker, New York, 1972.

11. I. Kaplansky, The homological dimension of a quotient field, Nagoya J. Math. 27 (1966), 139142.

12. S. Malik and J. I. Mott, Strong S-domains, J. Pure Appl. Algebra 28 (1983), 249-264.

13. M. Nagata, Local rings, Interscience, New York, 1962.

14. __ Finitely generated rings over a valuation domain, J. Math. Kyoto Univ. 5 (1966), 163169.

15. L. J. Ratliff, Jr., On quasi-unmixed local domains, the altitude formula, and the chain condition for prime ideals. II, Amer. J. Math. 92 (1970), 99-144.

Department of Mathematics, University of Tennessee, Knoxville, TenNESSEE 37996-1300 (Current address of D. F. Anderson, D. E. Dobbs and S. B. Mulay)

Département de Mathématiques, UniVersité de lyon I, 69622 Villeurbanne CEDEX, FranCE (Current address of Salah Kabbaj) 